Scuola di specializzazione in Endocrinologia e Malattie del ricambio della Università di Firenze Direttore: Prof. A. Lunedei

Istituto di Semeiotica Medica della Università di Firenze

Direttore incaricato: Prof. U. Teodori

\title{
NANISMO DEGENERATIVO TIPO DI AMSTERDAM \\ (Typus Amstelodamensis - Malattia di Cornelia De Lange) \\ PRESENTAZIONE DI UN CASO E CONSIDERAZIONI DI ORDINE GENETICO
}

\author{
di \\ A. Borghi, G. Giusti e U. Bigozzi
}

Recentemente è giunto alla nostra osservazione un raro caso di una sindrome degenerativa poco nota, che va sotto il nome di "tipo di Amsterdam ", o Malattia di Cornelia di Lange.

Descritta per la prima volta dalla De Lange nel 1933 in due bambine, questa entità morbosa è stata riconosciuta successivamente - sempre in soggetti di sesso femminile de Vedder (1933), De Lange (1938), Pincherle (1939), Julien-Marie e coll. (1946), Willemin-Clog e coll. (1947); non ci risulta che dopo tale data ne siano stati descritti altri casi.

La nostra osservazione presenta quindi un notevole interesse, non solo per la rarità della sindrone ( $9^{\circ}$ caso descritto nel mondo), ma anche e soprattutto per alcuni dati di ordine genetico, per la prima volta riscontrati, e che verranno successivamente esposti.

Il " tipo di Amsterdam " è caratterizzato dalle seguenti alterazioni, esaurientemente descritte dalla De Lange: sviluppo somatico e psichico enormemente ritardato; microbrachicefalia; sopracciglia folte e riunite sulla linea mediana, ciglia lunghissime; ipertricosi diffusa, più spiccata alla fronte e al labbro superiore; palato ogivale; prognatismo; mani piccole e tozze, con impianto del pollice più prossimale che di norma, e mignolo incurvato verso l'anulare (clinodattilia); micromelia omerale; impossibilità alla estensione completa del gomito; sindattilia bilaterale del $2^{\circ}$ e $3^{\circ}$ dito del piede; inoltre altre alterazioni malformative di minore entità, che possono anche mancare.

Come risulta dalla fotografia, e dall'esame obbiettivo della nostra piccola paziente, tutti i caratteri predetti sono presenti, e corrispondono perfettamente alle descrizioni più accurate degli altri AA. È da notare che nel nostro caso esistono alcune lievi discordanze rispetto al paradigma della De Lange (specialmente per quanto riguarda le mani), che sono forse da imputare alla età più avanzata della nostra paziente ( 6 anni); in effetti tutti i casi finora descritti, ad esclusione di quello di Vedder, riguardano soggetti di età compresa fra i 40 giorni e i due anni e mezzo.

Ricevuto il 6. VIII. 1954

25 - A. Ge. Me. Ge. (III) 


\section{Descrizione del caso}

O. G.; di anni 6; sesso femminile. Figlia unica.

Nata a termine da parto spontaneo; alla nascita era molto piccola: lunghezza $\mathrm{cm} .46$, peso $\mathrm{Kg}$. 1,5. Fu allattata artificialmente data la sua incapacità alla suzione del capezzolo, tuttavia si è sempre nutrita scarsamente. La crescita è stata molto lenta; a due anni misurava $50 \mathrm{~cm}$. di lunghezza; il peso non è conosciuto.

Alla nascita, ed anche per diversi anni successivi, presentava una notevole ipertricosi diffusa (che attualmente si è un po' attenuata) particolarmente spiccata alla fronte, al labbro superiore ed al tronco. L'inizio della deambulazione e la dentizione sono stati un po' ritardati rispetto alla norma. Fonazione quasi assente anche attualmente; soltanto da circa due mesi ha cominciato a pronunciare male qualche parola, ma senza senso; più che altro emette suoni inarticolati o brontolii. Ride frequentemente; conosce solo gli oggetti di uso più comune.

Fino a circa due mesi or sono non controllava gli sfinteri; orinava e defecava a letto, o dovunque si trovasse; attualmente riesce ad indicare, con suoni onomatopeici, le necessità corporali. Presenta mancinismo.

\section{EsAME OBBIETtivo:}

Notevole ipotrofia pondero-staturale (altezza cm. 103, peso Kg. 17,300). Muscolatura tonica e trofica, pannicolo adiposo scarso. Cute e mucose visibili ben sanguificate, apparato linfatico indenne.

Testa: micro-brachicefalia (diametro fronto-occipitale $\mathrm{cm}, 14,2$; bitemporale $13,5 \mathrm{~cm}$.) Globi oculari piuttosto grandi, con lievissimo esoftalmo, più distanziati che di norma. Ciglia molto lunghe; sopracciglia folte molto scure, che si riuniscono sulla linea mediana. Fronte con fine peluria, e poche vene evidenti. Capelli lunghi, grossi, lievemente ondulati. Naso piccolo, con narici volte in avanti. Labbro superiore molto prominente (tipo lattante), con peluria molto sviluppata; mento piccolo, angoli labiali lievemente stirati in basso. Orecchie grandi, inserite più in basso che di norma (orifizio del condotto uditivo esterno al si sotto del livello della apofisi zigomatica).

Bocca: sono presenti i denti della prima dentizione, in gran parte decalcificati e cariati. Lingua piuttosto grossa; palato nettamente ogivale; tonsille normali.

Tronco: posteriormente, si nota una notevole ipertricosi.

Niente di notevole a carico dei vari organi ed apparati interni.

Arti superiori: omero corto; impossibilità di estensione completa del gomito (angolo avambraccio-braccio circa $140^{\circ}$ ). Mani: dito medio molto lungo; mignolo incurvato verso l'anulare; pollice ed eminenza tenar inseriti più prossimalmente che di norma (i familiari affermano che questa particolarità era più evidente in passato; la bambina teneva inoltre, quasi in permanenza, il pollice racchiuso fra le altre dita).

Arti inferiori: normali, accenno di sindattilia (bilateralmente) alla base del $2^{\circ}$ e $3^{\circ}$ dito del piede. 
A. Borghi, G. Giusti e U. Bigozzi : Nanismo degenerativo tipo di Amsterdam, etc.

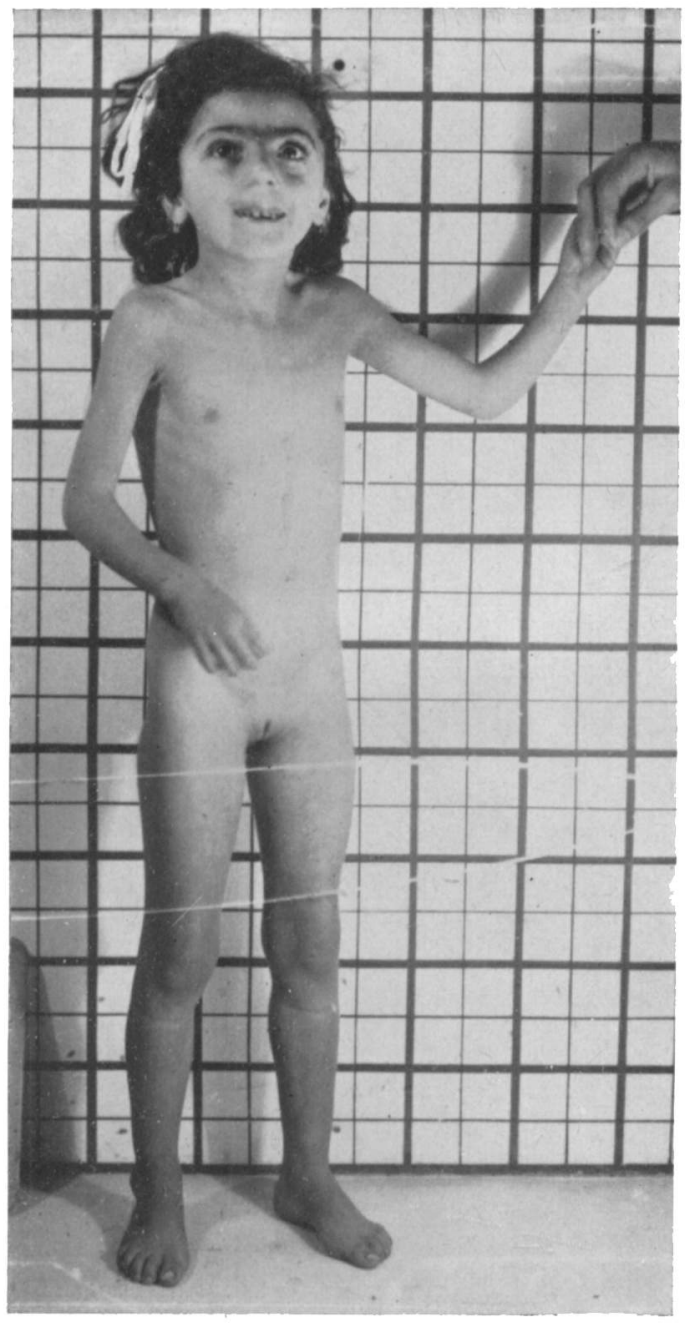

Fig. 1.

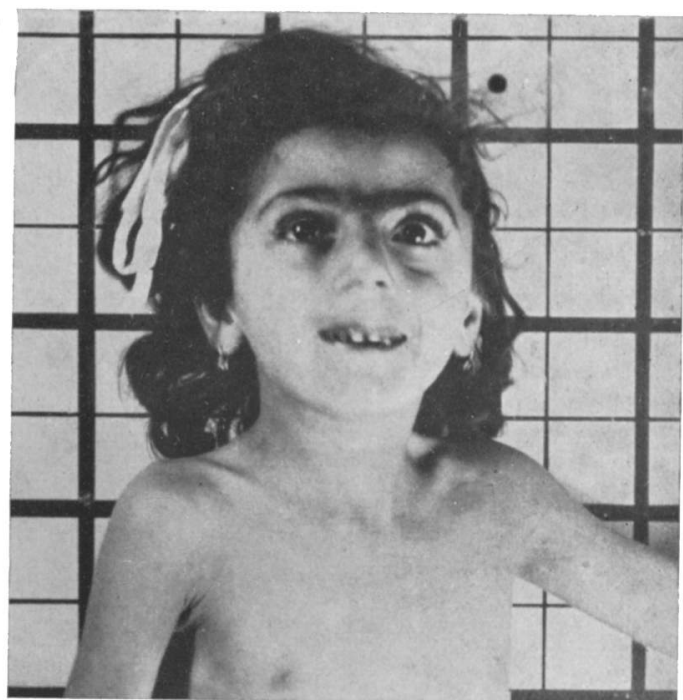

Fig. 2. Notare le soppracciglia folte riunite sulla linea mediana; le ciglia lunghe, le narici volte in avanti.

\section{EsAmi RadIOGRAFICI}

La radiografia dello scheletro mette in evidenza una ossificazione normale per l'età. La radiografia del cranio non permette di osservare alterazioni di sorta; anche la sella turcica si presenta completamente normale.

Radiografia delle mani: è evidente (vedi figura 3) l'incurvamento del mignolo verso l'anulare, ed una certa ipotrofia della III falange dello stesso dito. II $\mathrm{V}$ metacarpo sinistro presenta una al. terazione morfologica netta. $\grave{E}$ evidente

inoltre la sproporzionata lunghezza del medio, che, sebbene non descritta dagli AA., è rilevabile anche nei casi II e III della De Lange, ed in quello di Julien-Marie e coll.

Le ossa della mano non sono tozze come è descritto dagli altri AA.; ma i familiari - come pure le fotografie precedenti - testimoniano che le mani, in passato, erano molto più piccole e tozze di quanto non lo siano attualmente. 


\section{ESAMI DI LABORATORIO}

Gli esami da noi eseguiti sono ben pochi. Mentre abbiamo potuto procedere ad alcuni dosaggi ormonici nelle urine, l'opposizione dei genitori della bambina non ci ha permesso di eseguire l'esame emocromocitometrico, nè quelli ematochimici, che pure avrebbero avuto un notevole interesse. Comunque, $i$ dati in nostro possesso sono i seguenti:

R.W. (eseguita in passato) = negativa

Esame delle urine $=$ n.d.n.

17 - chetosteroidi urinari $=$ mgr. $1,35 / 24 \mathrm{~h}$.

Deidroidoandrosterone urinario $=\mathrm{mgr}$. $0 / 24 \mathrm{~h}$.

$17-21$ idrossi 20 -chetosteroidi $=$ mgr. $0,61 / 24 \mathrm{~h}$.

Fenolsteroidi = gamma $10 / 24 \mathrm{~h}$.

I valori dei dosaggi ormonali, eseguiti per la prima volta in questa entità morbosa, sono normali per l'età della paziente; ciò concorda con la osservazione della De Lange, che all'esame autoptico di un caso riscontrò, macro e microscopicamente, la completa normalità del sistema endocrino.

$$
\star \star \star
$$

I soggetti del «tipo di Amsterdam » finora descritti appartengono a famiglie apparentemente sane; se hanno fratelli, questi ultimi sono normali. Secondo la De Lange ed altri AA., si tratterebbe di una anomalia genotipica (non meglio precisata), per la cui manifestazione hanno notevole importanza anche i fattori ambientali; è da ricordare che nel caso di Julien-Marie e coll. vi era stato in effetti un tentativo di aborto al secondo mese.

Nel nostro caso, per la prima volta, esistono alcuni elementi che devono essere attentamente considerati, e che potrebbero far sospettare la esistenza di una influenza genetica sulla comparsa del tipo degenerativo di Amsterdam.

Innanzittutto, i genitori della bambina sono ambedue di razza ebraica; non sono parenti fra loro, ma nelle loro famiglie, per il passato, è stata largamente praticata l'endogamia. Come risulta dal grafico (fig. 4), devono essere presi in particolare considerazione i casi III 4 (madre), II 10 (nonna materna), III 1 (padre), II 6 (nonna paterna), e III 3 .

La madre presenta, fin da giovane, una labilità psichica estrinsecantesi con alternative di eccitazione e depressione; la situazione si è aggravata dopo la nascita della bambina. Non ha avuto nessuna malattia degna di nota; obbiettivamente non si riscontrano alterazioni a carico dei vari organi ed apparati. Ha avuto una sola gravidanza; per tre mesi dopo l'inizio di quest'ultima, ha avuto ugualmente le mestruazioni. Non desiderava di avere figli, e temeva di morire durante il parto.

La nonna materna presenta anch'essa alterazioni psichiche dello stesso tipo, aggravate negli ultimi tempi da fenomeni di arteriosclerosi cerebrale.

Il padre, alla nascita, pesava meno di $2 \mathrm{Kg}$.; è sempre stato molto gracile. Lo sviluppo è stato molto lento; la crisi puberale è avvenuta a 13 anni. Presenta criptorchidismo S. Obbiettivamente si osserva ipoevolutismo somatico (altezza $\mathrm{cm}$. 165; peso $\mathrm{Kg}$. 42), congiunto ad ipoevolutismo psichico. Cranio ristretto; modico esoftalmo; prognatismo e palato ogivale; gli incisivi ed i canini (superiori ed inferiori) sono disallineati ed embricati. 

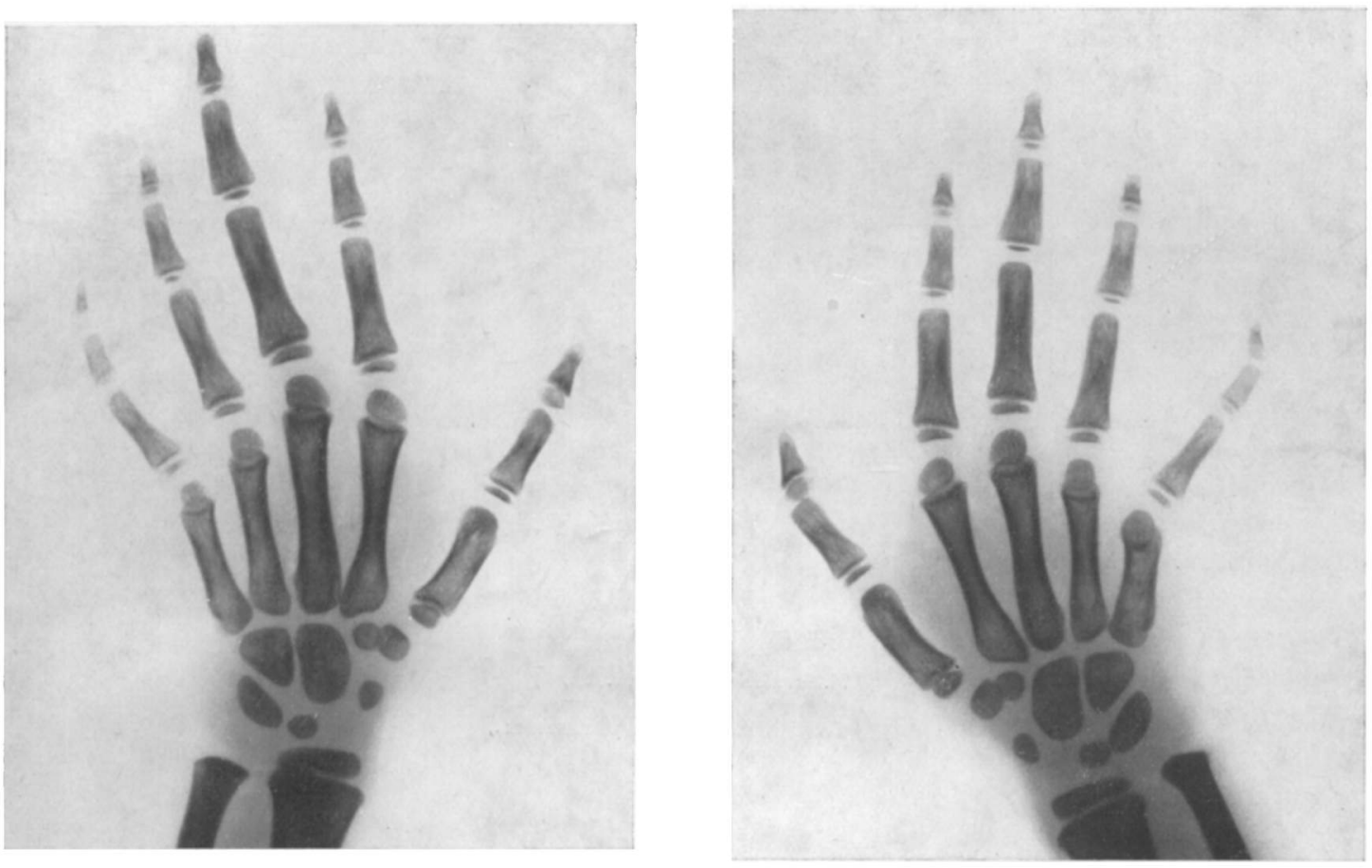

Fig. 3

La estensione del gomito è nettamente ridotta. Le mani sono allungate, con dito medio lungo, mignolo incurvato verso l'anulare, pollice ed eminenza tenar impiantati più prossimalmente che di norma. Le falangi presentano notevoli deviazioni dall'asse.

La nonna paterna - morta per malattia imprecisata - era, secondo le dichiarazioni dei familiari, il « ritratto " del figlio. Particolarmente notata la spiccata somiglianza delle mani ${ }^{1}$.

Assai più interessante è il soggetto III 3, i cui genitori, normali, erano cugini in I grado, in quanto figli di due sorelle della nonna del padre della nostra paziente (cfr. fig. 4). Questo soggetto fin dalla nascita presentava ipotrofia pondero-staturale, con ipertricosi diffusa, particolarmente spiccata al volto; sopracciglia molto marcate, narici volte in avanti, ipertrofia della lingua. Non ha mai parlato; emetteva solo grugniti, e le manifestazioni

1 È da notare che $\mathrm{i}$ dati genealogici sono stati forniti dai genitori della bambina, $\mathrm{i}$ quali ricordano con sufficiente precisione solo i dati concernenti fra i parenti più prossimi i quali sono compresi proprio tutti i casi con manifestazioni patologiche. Pertanto non si puó escludere la presenza di altri soggetti " anormali" nelle rispettive famiglie, dato che alcune alteraz. malformative, quali ad es. la clinodattilia, possono facilmente passare inosservate. 


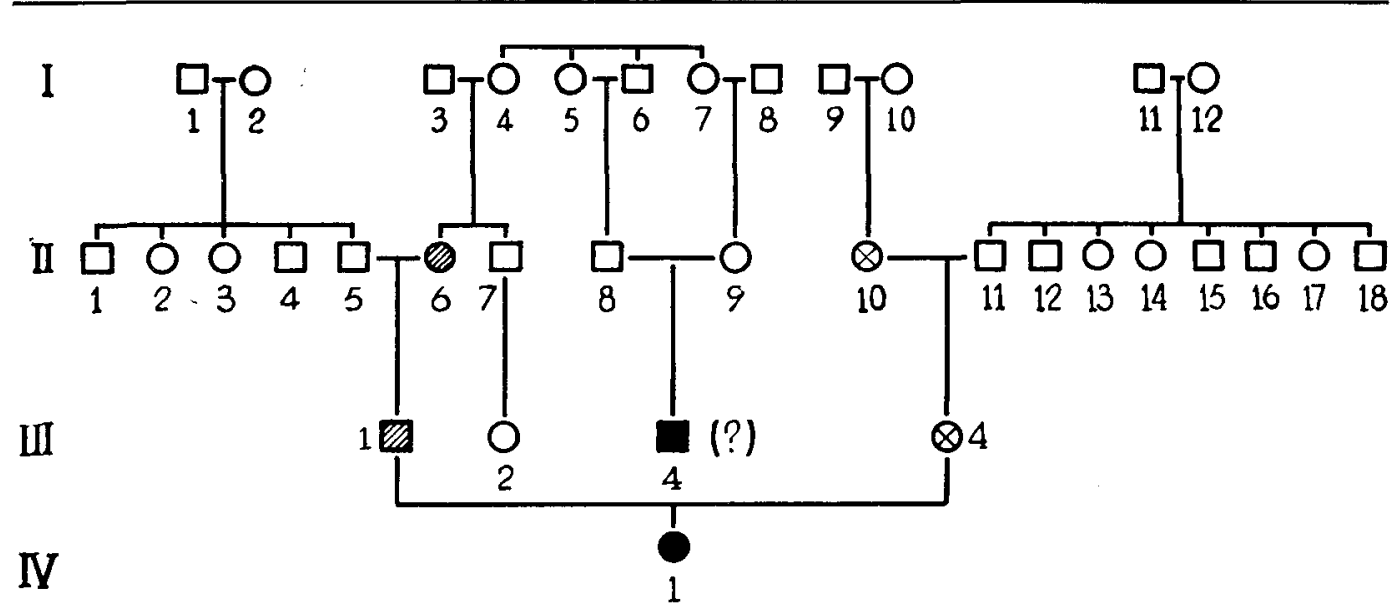

Tipo degenerativo di Amsterdam
Malformazioni varie (v. testo)
Costituzione "neuro-psicastenica,

Fig. 4

intellettuali erano quasi completamente assenti: presentava inoltre crisi convulsive. ̇̀ morto all'età di 15 anni per malattia imprecisata.

Purtroppo non siamo stati in grado di ottenere, almeno in visione, una fotografia di questo soggetto; ma dalla descrizione dei familiari si può sospettare con un certo fondamento che anch'egli appartenesse al c.d. "tipo di Amsterdam ", sebbene questa sindrome non sia stata finora descritta nel maschio. Comunque, anche se così non fosse, è certo che si tratta di alterazioni congenite di tipo degenerativo molto vicine, almeno in apparenza, a quelle della malattia di Cornelia De Lange. Anche le alterazioni degenerative presentate dal padre e dalla nonna paterna della bambina in esame si ritrovano tutte nel «tipo di Amsterdam ". Pertanto sembra giustificato domandarsi se alla base di questo particolare tipo di degenerazione multipla non siano dei fattori genetici, che solo in certe condizioni si rendono evidenti. Infatti è difficile pensare a pure influenze paratipiche, dato il gran numero di organi interessati; tra l'altro si deve tener presente che degli stimoli lesivi portati sull'embrione al II mese di vita intrauterina - come è avvenuto nel caso di Julien-Marie e coll, - ben difficilmente avrebbero potuto determinare alterazioni a così diversa localizzazione.

Ammettendo che il soggetto III 3 presentasse veramente un nanismo degenerativo di Amsterdam, si potrebbe pensare che questa alterazione sia legata a fattori recessivi; questa interpretazione sembrerebbe confermata oltre che dalla consanguineità dei genitori anche dal fatto che in tutti $i$ casi precedentemente descritti non esiste una familiarità della forma. 
La constatazione che il padre e la nonna paterna della probanda presentassero dei caratteri che possono essere considerati come microforme della stessa affezione può valorizzare anche l'ipotesi che questa sia determinata da fattori dominanti ad espressività variabile. (Si deve tener conto, fra l'altro, che il soggetto III 3 appartiene alla stessa linea familiare) Mentre non si può ammettere una dominanza con maggiore espressione (nella probanda) dovuta a una situazione di omozigotismo, data la mancanza assoluta di " microsintomi " nella madre; si potrebbe ammettere invece una dominanza con eterozigotismo, pensando a geni accessori trasmessi dalla madre. Il fatto che nella linea materna della bambina siano presenti elementi di una diatesi neuropsicastenica secondo Hofmeier potrebbe far pensare infatti alla collaborazione di altri fattori nella manifestazione della sindrome, date anche le note alterazioni del S.N.C. presenti nel "tipo di Amsterdam".

Infine, e con maggiore verosimiglianza, si potrebbe ammettere che nel caso di questa affezione ci si trovi di fronte ad un fenomeno di poligenia con presenza di uno o più geni dominanti, e di altri recessivi. È importante notare che la clinodattilia (presente nella probanda, nel padre e nella nonna paterna) è relativamente frequente, e si riscontra in numerose altre sindromi degenerative (mongolismo, ipertelorismo, ecc.). Essa può essere condizionata da un gene specifico, nel qual caso si ha trasmissione di questa sola anomalia, oppure può essere espressione di un pleiotropismo. Si potrebbe pensare quindi alla coesistenza di un gene dominante (clinodattilia) con altri recessivi, e con altri ancora, accessori.

Ma tutto questo rientra nel campo delle ipotesi, poichè nessuna conclusione può essere tratta dall'osservazione di un solo caso. È la prima volta infatti che può essere sospettata - con fondamento - una origine genetica dell'affezione, ma la modalità con cui questa si estrinseca è, per forza di cose, del tutto ignota.

Tuttavia la presenza di alterazioni degenerative, che soprattutto negli ascendenti della linea paterna sono dello stesso tipo - seppure attenuate — di quelle della sindrome di Cornelia De Lange, ci sembra confermare l'ipotesi di una influenza ereditaria, anzichè quella di una alterazione paratipica con difettoso sviluppo.

\section{Riassunto}

Gli AA. descrivono un nuovo caso di nanismo degenerativo di Amsterdam, che presenta tutte le caratteristiche fondamentali della sindrome.

L'indagine ormonologica, eseguita per la prima volta, ha dato valori normali (per l'età della paziente); ciò conferma la fondamentale normalità del sistema endocrino, riscontrata anatomo-patologicamente dalla De Lange.

Inoltre lo studio genealogico permette di far sospettare, per la prima volta, la esistenza di un fattore genetico nel determinismo di questo particolare tipo di degenerazione multipla. 


\section{Bibliografia}

De LANGe C.; Sur un nouveau type de dégéneration (Typus Amstelodamensis). Arch. de MId. des Enfants 36, 371, 1933.

DE LANGE C.; Nouvelle observation du "typus amstelodamensis ", et examen anatomo-pathologique de ce type. Arch. de Méd. des Enfants 41, 193, 1938.

Hofmeier K.; Die Bedeutung der Erbanlage für die Kinderheilkunde. F. Enke Verlag, Stüttgart 1938.

Jullen-Marie, Seringe P.; Cousin M.; Renaun C.; Typus Amstelodamensis. Le Nourrisson 34, 1 , 1946.

Pincherle B.; Première observation du typus amstelodamensis (De Lange) en Italie. Arch. de Méd. des Enfants 42, 443, 1939.

VedDer R.; Typus degenerativus amstelodamensis (De Lange). Nederl. Tijdschr. v. Geneeskunde 79, 993, 1935.

Willemin-Clog L.; Blanchet; Travade; Un nouveau cas de typus amstelodamensis. Coexistence d'une hypertrophie musculaire généralisée (Maladie de Cornélia De Lange). Arch. Franç. Pediatr. $4,100,1947$.

\section{RÉSUMÉ}

Les Auteurs décrivent un nouveau cas de «nanisme dégénératif de Amsterdam » qui présente toutes les caractéristiques fondamentales de cet syndrome. Le dosage des hormones, qui n'avait été jamais pratiqué jusqu'a maintenant, a donné des valeurs normales (étant donné l'âge du malade): ce qui est pour prouver que le systême endocrinien est fondalement normal, comme avai tété démontré du point de vue anatomo-pathologique par la recherche de $\mathrm{Ma}$ dame De Lange.

L’étude généalogique permet encore d'envisager - et cela pour la première fois - l'existence d'un facteur génétique qui détermine cette dégénération multiple, toute particulière.

\section{SUMMARY}

The authors describe a new case of degenerative nanism « typus amstelodamensis », which shows all fundamental characteristics of the syndrome. From the hormonic research, which was carried through for the first time, resulted normal values (for the age of patient); this fact proves the fundamental normality of the endocrine system, which was already found by De Lange in the anatomical-pathological field.

Moreover, the genealogical examination led for the first time to the assumption of the existence of a genetic factor causing this particular type of a multiple degeneration.

\section{ZUSAMMENFASSUNG}

Die Autoren beschreiben einen neuen Fall von degenerativem Zwergwuchs Typus Amstelodamensis der alle grundsätzlichen Merkmale des Syndroms aufweist. Die hormonale Untersuchung, die zum erstenmaldurchgeführt wurde, ergab (für das Alter der Patientin) normale Werte; diese Tatsache bestätigt die grundsätzlich Normalität des endrokrinen Systems, der schon De Lange im anatomisch-pathologischen $\mathrm{Be}$ reich begegnete.

Darüberhinaus führte die genealogische Untersuchung zum erstenmal zu der Annahme der Existenz eines genetischen Faktors, der diesen besonderen Typ einer multiplen Degeneration mitbestimmt. 